\title{
User studies of a sketch-based collaborative distant design solution in industrial context
}

\author{
Stéphane Safin, Pierre Leclercq \\ LUCID-ULg : Lab for User Cognition and Innovative Design, Faculty of Applied Sciences, \\ University of Liège, Belgium \\ stephane.safin@ulg.ac.be, pierre.leclercq@ulg.ac.be
}

\begin{abstract}
This study evaluates the opportunities and constraints linked to the technological transfer of a sketch-based distant collaborative environment, from academy to industry. The paper relates the concepts of the sketch-based collaboration, describes the Distant Collaborative Design Studio and proposes a methodology to assess the utility and usability of the system in two different companies. The results and conclusions show the issues linked to the implementation of such sketch-based collaborative environment in professional contexts.
\end{abstract}

Keywords: Sketch, creative design, collaborative design, virtual desktop.

\section{Introduction}

Since several years, the LUCID-ULg (Lab for User Cognition and Innovative Design) develops advanced CAD tools, in research and educational scopes. Among its most advanced prototypes, the DCDS (Distributed Collaborative Design Studio) is a sketch-based distant collaborative environment to support creative stages of design. This environment has already been tested and validated by the laboratory in an educational context. The objective of this study is to go beyond the walls of the university : the aim is to evaluate the opportunities of the technological transfer of this device in industrial domain, which is quite different from academic and research contexts, and to evaluate the core issues linked to the sketch-based collaboration in professional practices.

This paper describes the INNOVATIC project, a validation study of the DCDS technological transfer from university to industry. From field studies, anchored in real professional contexts, we drive conclusions about the utility of the DCDS, and show how our prototype should be enhanced to respond to true demands of industrial contexts. This study is grounded in a more general context of a user-centered methodology, which has prevailed from the very beginning of the DCDS development.

At first, we briefly describe the context of the project, i.e. the usefulness of sketchbased collaboration in the early steps of design. We then give a description of the DCDS environment. The methodology frame of our technological transfer study and 
its results are given in the next two sections. We then conclude on perspectives about the utility of such an approach and the issues linked with sketch-based collaborative design in industrial context.

\section{Context}

In a wide range of activity sectors, collaboration has been intensified, notably in the design domains. Collective work is increasingly organized simultaneously (rather than sequentially as it used to be in the past). Moreover, design teams are often geographically distributed, and the need for distant real-time interaction is consequently emerging. A lot of effective systems are available for sharing information, but most of them are asynchronous (e.g. database server, email...) or allow only partial interaction (e.g. phone or visioconference).

Virtual reality is a promising way to respond to challenges in organizations and processes. The LUCID-ULg proposes a system for sketch-based multimodal interaction, which is based on the invisible computer paradigm [1]. Instead of requiring designers to change their way of conceiving, we propose to support one of the most usual way of collaborating : the free-hand sketching, which plays an crucial role, especially during initial stages of design. Even in domains where design constitutes only a part of the whole process (as for instance building or naval engineering, architecture, industrial design or town planning), there are great ideas that emerge from quick drawings made on a napkin! Many authors grant to the upstream sketching phase the biggest magnitude : it reduces the cognitive charge, makes designers explore more solutions, enhances creativity, and eases the artifact communication [2].

\section{DCDS}

Our prototype, named Distributed Collaborative Design Studio (DCDS) is composed of a hardware part - the Design Virtual Desktop - and a software part - SketSha (for sketch sharing), completed by external modules.

The Design Virtual Desktop (fig 1) consists of an electronic A0 table with a suspended ceiling equipped with a projection system offering a large working surface (approximately $150 \times 60 \mathrm{~cm}^{2}$ ). An electronic stylus allows the drawing of virtual sketches onto this surface. The central unit is located in the ceiling. This leaves the stylus as the only interaction tool, so that the computer can disappears from designers' mind.

The SkeSha software (fig. 2) is a shared drawing environment allowing several virtual desktops to be connected to the same drawing space. Various functionalities, such as a panel of colored pens (and an eraser) and a navigation widget (zoom, translate, rotate), are proposed through intuitive graphical widgets. This software captures the strokes that compose the sketch, share them between the different distant locations (through a classic internet connection) and transmits the whole information in real-time on the active boards through video-data projectors. 


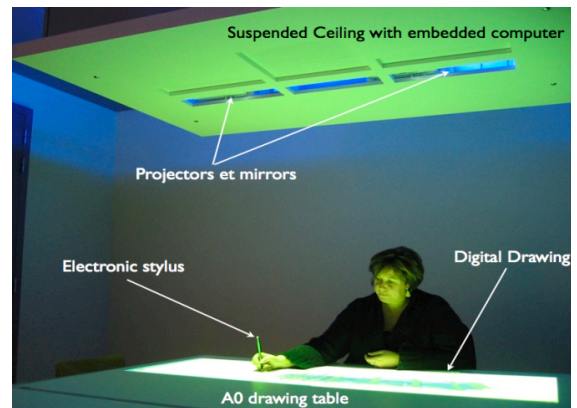

Fig 1 : Virtual Desktop.

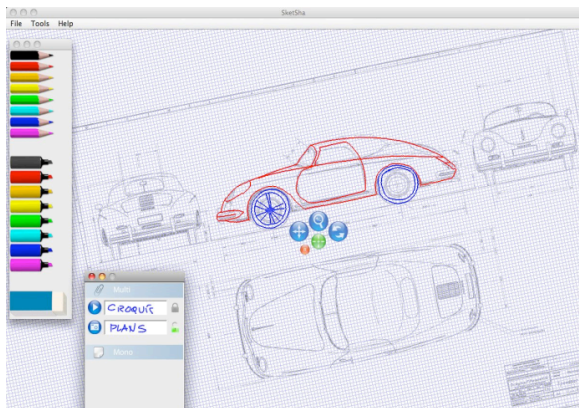

Fig. 2 : SketSha Interface.

Some layout facilities have also been included in the prototype, such as the possibility to draw and to manage different sheets of virtual paper, to delete or duplicate them, and to manage their transparency. The software also allows to import CAD plans and bitmap images.

Pointing, annotating and drawing are possible thanks to the electronic pen that activates the virtual desktop drawing recognition. Social exchanges are transmitted through external modules (videoconference commercial solutions) in order to support the vocal, the visual and the gestural aspects of the collaboration. The system is thus completed by a 24 inches display with an integrated camera, that allow the participants to see and talk to each others, in an almost $1 / 1$ scale, during a real-time conference. This integrated camera is in fact a very simple way to avoid the gaze deviation when talking to interlocutor(s) (see fig 3 for the whole environment).

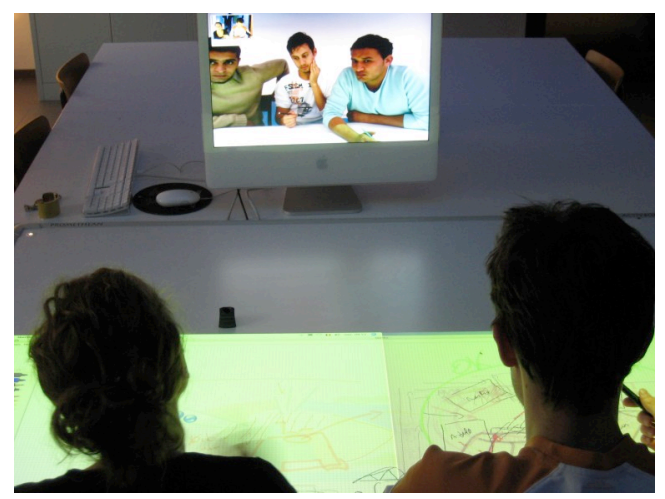

Fig. 3 : Distributed Collaboration Design Studio.

Initially thought specifically for architecture, our system revealed itself to be useful for many other design domains.

Following the user-centered framework underlying the DCDS development, the system has been first tested in different sessions: individual uses [3,4], distant collaborative sessions $[5,6]$ and pedagogical long duration collaborative work settings $[7,8,9]$. 
All testing sessions have been videotaped (fig 4) and analyzed by ergonomists. They have demonstrated the simplicity of the system, which is quickly mastered by all participants. They have also shown the richness of interactions in our environment, and the system ability to support collaboration close to co-present situations. The long-term sessions have also shown its utility as an efficient cooperation tool for concrete projects.

Furthermore, industrial partners (mechanical engineering office and an architectural office) have shown to be very interested by this environment for their professional practice and foresee many real advantages by adopting our technological solution.

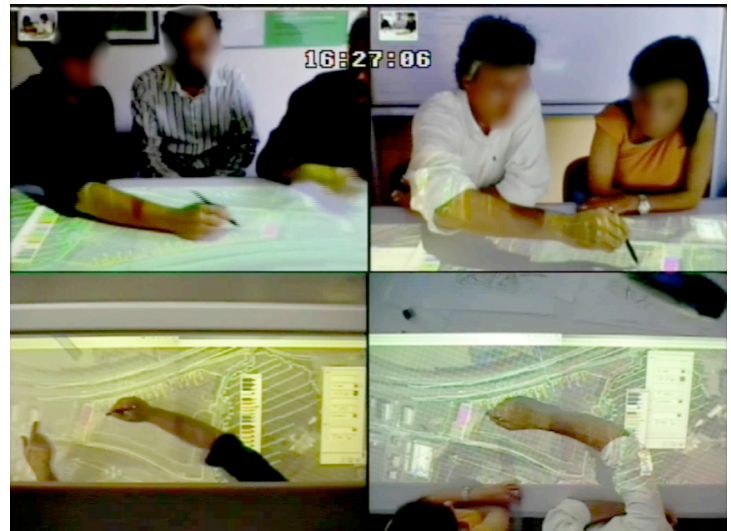

Fig. 4 : Screenshot of collaborative session recording (front view and top view).

\section{Methodology}

In order to deepen the user-centered approach, we needed to assess the opportunity of the DCDS technological transfer to industrial context. With this objective we designed a three steps methodology for involving users in the reflection.

1 - On-site interviews to understand the collaborative habits of companies : the idea is to identify the opportunities of the introduction of the DCDS, as well as challenges and issues it raises, from an organizational point of view.

2 - Demonstration of the prototype, composed of three steps : a formal demonstration leaded by the researchers; an artificial situation designed to let people try the system ; and a structured brainstorming, aiming at identifying the opportunity of using such a system

3 - Real work session : one half-day work session has been planned to become a part of a real project of the users. It consisted in a real meeting with different actors of a same design project but, instead of taking place around a table in the office, it took place on two connected DCDS, which were located in two different rooms of the University of Liège.

The first two steps were targeting company directors and managers, who are aware of the organizational challenges linked to the introduction of such a system. The last step was targeting the main designers, who are potential end users of the device. 
Therefore, the first two steps aimed at identifying utility issues, while the last one was more linked to the system's usability.

This three steps methodology has been implemented with two sets of users : in a large international architecture office (about 120 collaborators in 3 countries) and a mechanical engineering office of about 60 people. These two kinds of company have different types of organization, different habits, different legal steps in design process, and above all different uses of free-hand sketches. The first step gathered 4 users in architecture office and 4 in industrial design office, the second step 3 and 2, and the real work sessions involved each time 4 users, distributed on two DCDS.

All the steps have been recorded and/or videotaped for analysis purpose. The figure 4 shows a screenshot of the recording of the third phase work sessions.

\section{Results}

This study has led to two sets of results. The first one is related to current collaboration practices and the way they could be enhanced or, at least, modified by the DCDS. They refer to the utility of the system. The second set of results is complementary : it gives insights for identification of usability problems and missing functions, through the analysis of activities. The following sections summarize the main observations and conclusions.

\subsection{Envisioned utility of the system and organizational changes}

In the mechanical engineering office, every project goes through systematical steps, being always the same. 1) At first, there is an encounter with the client in a copresent meeting (in the company or in the client's place) where all initial information is exchanged. Then the first working stage is leaded inside the company. Several workers are already involved, and share numerous documents. Using the DCDS in a copresent situation should be interesting for their project meetings, because it can keep track of the annotations and modification, and share them among every actors. 2) Afterwards the collaboration with the client intensifies. Many documents, plans etc. are exchanged and annotated. For this purpose, the DCDS could be a beneficial tool to save time, especially when the client is far from the company. 3) Then loops of "internal work/work with the client" continue until the final presentation. All these exchanges are usually asynchronous, which may lead to losses of time and misunderstandings. The real-time capacity of the DCDS is therefore highlighted by the interviewees. Furthermore, they emphasis reduction of paper and travels, and limitation of the ecological footprint.

In the observed architecture office, the workflow is well structured : a project starts by a design phase for a few weeks or a few months, before going in a development phase. These two stages are clearly distinct in the organizational structure of the company. The design cell uses its own tools and standards. It is composed by people with different qualification than those of the development team and is mainly leaded by time constraints : the work organization has to be very flexible. Pen and paper 
sketches are core tools for the preliminary design, as well as some computer graphics tools. The main issue of the development cell is related to the harmonization of representations. Due to the length of projects (generally several years, up to seven), the production has to be standardized, to manage regular turnover of workers. CAD tools are mostly used by this development cell.

For the architects, the DCDS offers several advantages. It is considered as a "quite obvious necessity", as it allows reducing displacements in every project step.

- For the design stage, it allows to communicate between different designers, in real-time and using a simple manner. This will lead to an interesting change in the power structure of the company. For the moment, the head office leads the design process and the distant partners feel sometimes disempowered from the core design. They are just involved in a few meetings. Having the possibility to make day-to-day distant meetings should enhance the investment of distant partners in the design, with a better balance of teams responsibilities.

- For the development phase, the DCDS allows a quick communication between the architects and the contractors on the building site, enabling quick problem solving, mainly based on plans annotations.

- It also facilitates communication with the client, who is often far from the head office. For this last utility, however, 3D representations could still enhance the DCDS offer.

For the two situations, the main problem is linked to the "heaviness" of the hardware infrastructure. The system is still costly and bulky, especially to be installed in small branch offices, or at the client's office. Furthermore, for the design phase, the architects fear that the introduction of such a system lead to the multiplication of short meetings, which are perhaps not always necessary. Without a strong organizational regulation, this may globally lead to a loss of time.

\subsection{Usability issues and needed functions}

The testing in the two situations (with the mechanical engineering office and the architecture office) was qualified as a success. Real meetings took place and the results were very satisfying for both teams. This testing has led to several conclusions.

At first, it has to be noted that some usability issues need improvement : difficulties with layers management, calibration problems and lack of the undo function are highlighted. Nevertheless, these adjustments are qualified as minor by the development team.

The major strength of the DCDS is linked to the "natural" interaction modalities and the completeness of collaborative interactions between distant users. Multimodality of interaction (sketches, annotations, voice, images, gestures) is appreciated and several suggestions are linked to an enhancement of the offered simplicity : facilitating the pointing, word and gesture recognition, gestural interface are proposed. On a global pointy of view, it has been noted that the real-time collaboration allowed by the system leads to an enhancement of participation and a time profit for generating and validating ideas.

Other suggestions are linked to the management of collaboration inside the system : users would like to clearly identify who is drawing at each time and which strokes 
are drawn by which user. In the same idea, they would like to implement a digital signature. Indeed, until now sketches are drawn on the DCDS with no legal value. Electronic signatures should allow real legal collaboration. The system should also allow the management of different project versions that may be numerous. Finally, some users proposed to use the system to keep track of the collaborative process, by audio recordings for instance : the drawing could then be doubled by verbal annotations.

One of the major propositions, already evoked in the previous section, is linked to the availability of a mobile version of the device. More generally, the users claim for a more flexible working surface : some users want the drawing surface larger, other ones consider the virtual desktop as already too large for their use. This flexibility implies obviously reflections about software compatibility between different devices, and especially management of several drawing surfaces of different sizes into the real-time collaboration space.

The import-export modules should be enhanced. For the moment they lack to support heavy files, which are numerous in the large projects usually involving distant collaboration. Moreover, designers of the mechanical engineering office (who are less used to free-hand drawing than the architects) would really benefit to using vectorial drawings.

The issue of transfer security is also an important point, because many information exchanged in the design are confidential by nature. DCDS should be enhanced to avoid security failures.

Compatibility with "classical" CAD tools is also highlighted. The system should support different file formats and should allow a direct link with the other tools used by companies.

Finally, the Internet stability seems satisfying. SketSha is quite stable for long-term sessions. The main problem of Internet transfer are linked to the common visioconference module.

\section{Perspectives}

The results have led to several conclusions. At first it is interesting to notice that the DCDS seems to be appropriate in two quite different contexts, namely in architectural design and in mechanical engineering design. If these two contexts imply different issues and development propositions, they support quite similar activities. They can be classified into 4 categories : (1) naturalness of interaction, (2) collaborative process and legal and organizational issues, (3) compatibility with other tools and (4) flexibility of hardware component and mobility. From this point specific modules should be differentiated, according to the different contexts. For instance, the use of vectorial drawing is a core issue for the mechanical engineering office, but not for the architecture company.

The study and its methodology, which are proposed here to validate the technological transfer, are simple but seems efficient : thanks to a few working sessions, it allows to identify a lot of issues and potential developments. We argue this is only possible throughout user studies in real settings. This study also reinforces 
our industrial partnerships, by demonstrating to our partners and potential clients the effective utility of our technological proposition.

Finally we have to highlight that collaboration is not just a matter of collaborating. It raises several issues linked to organization, management, legal aspects, transfers security, etc. All these issues have to be taken in account when designing appropriate technologies for collaboration. The simple technological capabilities and the "smart aspects" of a new tool are not sufficient : collaboration is rooted in organizations and habits that need to be taken into account. Our next steps will be to test the DCDS in long-term settings inside the companies. This will show us deeply the issues linked to organizational constraints and advantages of its usage. This will also be the opportunity to gather a lot of complementary information about the usability and the potentialities of our DCDS system.

\section{References}

1. Norman, D.A. (1998). The invisible computer, MIT Press, Cambridge University Press, MA

2. Cross N. : Strategies for Product Design, Third Edition. The open University, Milton Keynes, UK, Wiley (Eds) (2000).

3. Safin, S., Boulanger, C. \& Leclercq, P (2005). A Virtual Desktop's First evaluation for an Augmented Design Process. Proceedings of Virtual Concept 2005. Biarritz, France, November

4. Safin, S., Leclercq, P. \& Decortis, F. (2007) Impact d'un environnement d'esquisses virtuelles et d'un modèle 3D précoce sur l'activité de conception architecturale. Revue d'Interaction Homme-Machine. Vol 8, N², pp. 65-98

5. Burkhardt, J-M., Détienne, F., Moutsingua-Mpaga, L., Perron, L., Safin, S. Leclercq, P. (2008a) Multimodal collaborative activity among architectural designers using an augmented desktop at distance or in collocation. Proceedings of ECCE 2008: European Conference on Cognitive Ergonomics. 16-19 September - Madeira, Portugal.

6. Burkhardt, J-M., Détienne, F., Moutsingua-Mpaga, L., Perron, L., Safin, S. Leclercq, P. (in press) Conception architecturale collaborative avec un «bureau augmenté »: une étude exploratoire de l'effet de la distance et de la co-localisation. Actes de SELF 2008 : 43ème congrès de la Société d'Ergonomie de Langue Française. 17-19 septembre 2008 - Ajaccio

7. Elsen, C. \& Leclercq, P. (2008) SketSha" or the sketch power to support collaborative design Proceedings of CDVE : 21-25 September, Majorque

8. Kubicki S., Bignon J-C., Lotz J., Gilles Halin G., Elsen C. \& Leclercq P., 2008. Digital Cooperative Studio. ICE 2008 14th International Conference on Concurrent Enterprising, Special session ICT-supported Cooperative Design in Education, Lisboa, Espagne.

9. Kubicki S., Bignon J-C., Gilles Halin G., \& Leclercq P., 2008. Cooperative Digital Studio IT-supported cooperation for AEC students. CIB-W78 25th International Conference on Information Technology in Construction, Santiago de Chile, Chili. 